\title{
Erratum
}

\section{Notch1 in primary effusion lymphoma: a clinicopathological study}

Huan-You Wang, Franklin S Fuda, Weina Chen and Nitin J Karandikar

Department of Pathology, Dallas, TX, USA

Modern Pathology (2010) 23, 1043; doi:10.1038/modpathol.2010.79

Correction to: Modern Pathology (2010) 23, 773-780;

doi:10.1038/modpathol.2010.67

In the above article, the authors' affiliation was incomplete; the complete affiliation is listed below:

Department of Pathology, UT Southwestern Medical Center, Dallas, TX, USA 